\title{
COVID-19 and pulmonary hypertension
}

\section{Posted May 1, 2020}

\section{ABSTRACT}

Pulmonary hypertension $(\mathrm{PH})$ is a pulmonary vascular disease characterized by pulmonary arterial remodeling and vasoconstriction leading to elevated pulmonary artery pressure and, ultimately, right heart failure. So far, few cases of COVID-19 disease in patients with PH have been reported. Caution is warranted in interpreting this observation as data are evolving and several factors may influence the number of reported cases of PH and COVID-19. Social distancing and quarantine could play a role, especially for patients with chronic diseases who might be more vigilant of their potential for respiratory infection. In addition, PH is a rare disease, and because testing is not universal, we could be underestimating the number of cases.

Other hypothetical factors to consider are the underlying pathophysiology of $\mathrm{PH}$ and the medications used to treat $\mathrm{PH}$ and their implications in COVID-19.

\section{INTRODUCTION}

Patients with underlying chronic health conditions, mainly hypertension and cardiovascular disease, are at increased risk of developing more severe COVID-19 disease. Pulmonary hypertension (PH) is a pulmonary vascular disease characterized by pulmonary arterial remodeling and vasoconstriction leading to elevated pulmonary artery pressure and, ultimately, right heart failure.

So far, there have been only a few reported cases of COVID-19 disease in patients with PH. One should be cautious when interpreting this observation. As the pandemic is still unveiling, data sharing and publication might be skewed and inaccurate. And there could be other important factors playing a role in the number of reported cases of PH and COVID-19.

The implementation of social distancing and quarantine could play a role, especially for patients with

\footnotetext{
The statements and opinions expressed in COVID-19 Curbside Consults are based on experience and the available literature as of the date posted. While we try to regularly update this content, any offered recommendations cannot be substituted for the clinical judgment of clinicians caring for individual patients.

doi:10.3949/ccjm.87a.ccc021
}

chronic diseases who might be more vigilant of their potential for respiratory infection. In addition, $\mathrm{PH}$ is a rare disease, and because testing is not universal, we could be underestimating the number of cases. Other hypothetical factors to consider are the underlying pathophysiology of $\mathrm{PH}$ and the medications used to treat PH and their implications in COVID-19.

\section{PATHOPHYSIOLOGY}

Coronaviruses are a large family of viruses that range from the common cold to the severe acute respiratory syndrome (SARS-CoV). During the SARSCoV epidemic in 2003, and a lot of work was put into investigating effective therapies. Of the different pathways investigated, the arginine-nitric oxide $(\mathrm{NO})$ and renin-angiotensin pathways are of interest in both $\mathrm{PH}$ and coronavirus diseases. The angiotensin-converting enzyme 2 (ACE2), a member of the vasoprotective axis of the renin-angiotensin system (RAS), was recognized as a functional receptor for coronavirus on epithelial cells, identifying a role for ACE2 in coronavirus cell entry. ${ }^{1}$ In addition, ACE2 catalyzes the conversion of angiotensin II to angiotensin (1-7), and there is an abundance of evidence supporting a protective role for ACE2 in a variety of lung diseases, even proposing ACE2 supplements to reverse lung pathology. ${ }^{2}$

Another member of RAS is angiotensin II, which has adverse effects in the lungs that contribute to injury and inflammation. A consideration in COVID19 is decreased downregulation of angiotensin II and low angiotensin levels leading to increased pulmonary vasoconstriction and dysregulation of hypoxic vasoconstrictive mechanisms. It is well recognized that the activation of RAS is involved in the development of $\mathrm{PH}$, with reduced ACE2 levels in patients with PH. Recombinant ACE2, pulmonary overexpression of ACE2, and the use of small-molecule ACE2 activators were shown to attenuate $\mathrm{PH}$ through increased production of angiotensin (1-7) and endothelial NO synthase-derived $\mathrm{NO}$ bioavailability. ${ }^{3}$ It is unclear 
whether reduced ACE2 in $\mathrm{PH}$ would be protective or could promote lung injury in COVID-19 disease.

Nitric oxide plays an important role in the pathogenesis of $\mathrm{PH}$, and therapies targeting the $\mathrm{NO}$ pathway have been used successfully in treating PH. During the 2003 SARS epidemic, inhaled NO was shown to have antiviral activity against the coronavirus. Inhaled $\mathrm{NO}$ reversed $\mathrm{PH}$, improved severe hypoxia, and shortened the length of ventilator support compared to matched control patients with SARS-CoV. ${ }^{4}$ In-vitro studies showed that $\mathrm{NO}$ donors increased the survival rate of SARS-CoV-infected eukaryotic cells, suggesting direct antiviral effects of NO.,6 Since SARS-CoV shares most of the genome of the new coronavirus SARS-CoV-2, there could be a potential benefit for inhaled NO therapy in COVID-19 patients. As such, lower NO levels in $\mathrm{PH}$ patients could put them at a disadvantage. At the same time, it is unclear what effects therapies that enhance the NO pathway could have in patients with COVID-19.

Endothelin-1 is upregulated in $\mathrm{PH}$, and therapies targeting the endothelin pathway, mainly endothelin-receptor antagonists, are used extensively to treat PH. Studies have shown cross-talk between the endothelin system and RAS. In fact, endothelin-1 can downregulate ACE-2 expression in lung epithelial cells, and endothelin-receptor antagonists inhibit the angiotensin II induced vasoconstriction. ${ }^{7,8}$ Others showed that angiotensin (1-7) attenuates the actions of endothelin-1 on endothelial cells, mainly inflammation and growth. ${ }^{9}$ The data so far are hypothesis generating; however, as we learn more about the new coronavirus with ongoing and future studies, our understanding of the link between COVID-19 and $\mathrm{PH}$ pathophysiology and medical therapy will expand.

\section{- CLINICAL ASPECT}

Our knowledge of this new coronavirus is expanding, although most studies and published data are descriptive and exploratory. We know that COVID19 causes more severe disease in patients with comorbidities, especially cardiovascular diseases. Thus, patients with PH should follow the CDC guidelines for people at high risk by staying at home as much as possible, avoiding crowds, and maintaining at least a 6 -foot distance from others. They should wash their hands frequently and avoid touching their faces. The $\mathrm{CDC}$ also recommends covering the mouth and nose with a cloth when around others and when out in a public place. Patients should continue their current PH medications and maintain enough medications for several weeks.
If a patient with $\mathrm{PH}$ develops COVID-19, the PH medications should not be interrupted or changed unless advised by the primary PH provider. For severe respiratory infections that require intensive care unit (ICU) admission, patients should be treated whenever possible at tertiary centers capable of providing medical therapy, extracorporeal life support, and advanced treatment, including lung transplantation. Patients with $\mathrm{PH}$ can deteriorate rapidly with right ventricular (RV) decompensation and failure in the setting of hypoxemia and inflammatory cytokine release. ICU treatment of $\mathrm{PH}$ patients with COVID-19 includes treatment of the underlying viral respiratory infection, supportive measures including ventilator support (if required), meticulous fluid management to optimize RV preload, reduction of RV afterload with pulmonary vasodilators, and an individualized use of inotropes and vasopressors. ${ }^{10}$

In addition to receiving medical therapy for COVID-19 and for the associated cytokine storm, attention should be given to treating hypoxia. Supplemental oxygen is provided to maintain oxygen saturation above 90\%. Intubation should be avoided, if possible, as positive pressure ventilation increases intrathoracic pressure impeding RV preload and sedatives affect cardiac function and cause vasodilation leading to systemic hypotension and hemodynamic collapse. If the patient requires intubation, vasoactive drugs should be started before anesthesia. Etomidate is the preferred drug for induction of general anesthesia as it has little effect on cardiac contractility and vascular tone. ${ }^{11,12}$ Hypotension needs to be aggressively treated with systemic vasopressors to avoid decreased RV coronary perfusion and loss of the contribution of the interventricular septum to RV ejection. Sedation is maintained using opioids with benzodiazepines or propofol. Ventilator settings should aim to keep airway pressures to a minimum with low tidal volumes and moderate positive end-expiratory pressure while ensuring adequate oxygenation and ventilation.

Meticulous fluid management, reducing venous filling pressures, and normalizing cardiac output are essential in managing these patients. Fluid management is challenging as both hypovolemia and hypervolemia can have detrimental effects on blood pressure, organ perfusion, and cardiac function. In most cases, there is fluid overload, and this negative fluid balance should be targeted through diuresis or even ultrafiltration. However, if hypovolemia is suspected, judicious fluid administration should be attempted as hypovolemia may worsen cardiac output.

Another important consideration is reducing RV 
afterload through the use of pulmonary vasodilators. Point-of-care cardiac ultrasound and invasive hemodynamic monitoring (Swan-Ganz catheterization) may be useful. Inhaled $\mathrm{NO}$ or prostacyclin can be added to patients already on pulmonary vasodilators. Inhaled pulmonary vasodilators also are used as rescue therapy in refractory acute respiratory distress syndrome. They dilate pulmonary arterioles in ventilated lung units improving oxygenation through better ventilation-perfusion matching and reducing pulmonary vascular resistance. Inhaled $\mathrm{NO}$ should be preferentially considered in COVID-19 in view of its additional antiviral effects. To further improve cardiac output, inotropes including dobutamine or other alternatives such as phosphodiesterase-3 inhibitors or calcium sensitizing agents should be considered. If needed, add vasopressors to ensure an adequate perfusion pressure..$^{13,14}$

\section{REFERENCES}

1. Li W, Moore MJ, Vasilieva N, et al. Angiotensin-converting enzyme 2 is a functional receptor for the SARS coronavirus. Nature 2003; 426(6965):450-454. doi: 10.1038/nature02145

2. Jia H. Pulmonary angiotensin-converting enzyme 2 (ACE2) and inflammatory lung disease. Shock 2016; 46(3):239-248. doi: 10.1097/ SHK.0000000000000633

3. Zhang J, Dong J, Martin $\mathbf{M}$, et al. AMP-activated protein kinase phosphorylation of angiotensin-converting enzyme 2 in endothelium mitigates pulmonary hypertension. Am J Respir Crit Care Med 2018; 198(4):509-520. doi: 10.1164/rccm.201712-25700C
4. Chen L, Liu P, Gao H, et al. Inhalation of nitric oxide in the treatment of severe acute respiratory syndrome: a rescue trial in Beijing. Clin Infect Dis 2004; 39(10):1531-1535. doi: 10.1086/425357

5. Keyaerts E, Vijgen L, Chen L, et al. Inhibition of SARS-coronavirus infection in vitro by S-nitroso-N-acetylpenicillamine, a nitric oxide donor compound. Int J Infect Dis 2004; 8(4):223-226. doi: 10.1016/j. ijid.2004.04.012

6. Akerstrom S, Mousavi-Jazi M, Klingstrom J, Leijon M, Lundkvist A, Mirazimi A. Nitric oxide inhibits the replication cycle of severe acute respiratory syndrome coronavirus. J Virol 2005; 79(3):1966-1969. doi: 10.1128/JVI.79.3.1966-1969.2005

7. Zhang H, Li Y, Zeng Y, et al. Endothelin-1 downregulates angiotensin-converting enzyme-2 expression in human bronchial epithelial cells. Pharmacology 2013; 91(3):297-304. doi: 10.1002/ajh.24269

8. Wenzel RR, Ruthemann J, Bruck H, et al. Endothelin-A receptor antagonist inhibits angiotensin II and noradrenaline in man. Br J Clin Pharmacol 2001; 52(2):151-157. doi: 10.1046/j.0306-5251.2001.01422.x

9. Yusuf $\mathbf{H}$, Montezano AC, Callera GE, et al. Angiotensin 1-7 attenuates endothelin-1-induced endothelial cell inflammation and growth through nitric oxide production and activation of mas and endothelinb receptors. Hypertension 2018; 60(suppl 1):Abstract 258.

10. Jentzer JC, Mathier MA. Pulmonary hypertension in the intensive care unit. J Intensive Care Med 2016; 31(6):369-385. doi: 10.1177/0885066615583652

11. Gordon C, Collard CD, Pan W. Intraoperative management of pulmonary hypertension and associated right heart failure. Curr Opin Anaesthesiol 2010; 23:49-56. doi: 10.1097/ACO.0b013e3283346c51

12. Pritts CD, Pearl RG. Anesthesia for patients with pulmonary hypertension. Curr Opin Anaesthesiol 2010;23(3):411-416. doi: 10.1097/ ACO.0b013e32833953fb

13. Hoeper MM, Granton J. Intensive care unit management of patients with severe pulmonary hypertension and right heart failure. Am J Respir Crit Care Med 2011; 184(10):1114-1124. doi: 10.1164/ rccm.201104-0662Cl

14. Grinstein J, Gomberg-Maitland M. Management of pulmonary hypertension and right heart failure in the intensive care unit. Curr Hypertens Rep 2015; 17(5):32. doi: 10.1007/s11906-015-0547-z 